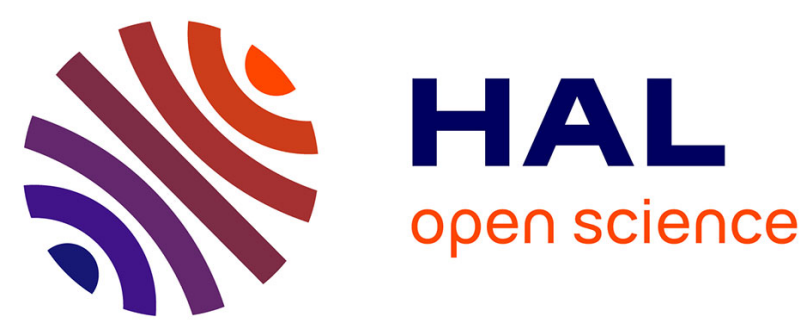

\title{
Colonization pattern of coagulase-negative staphylococci in preterm neonates and the relation to bacteremia
}

\author{
M. Björkqvist, M. Liljedahl, J. Zimmermann, J. Schollin, B. Söderquist
}

\section{To cite this version:}

M. Björkqvist, M. Liljedahl, J. Zimmermann, J. Schollin, B. Söderquist. Colonization pattern of coagulase-negative staphylococci in preterm neonates and the relation to bacteremia. European Journal of Clinical Microbiology and Infectious Diseases, 2010, 29 (9), pp.1085-1093. 10.1007/s10096-0100966-3 . hal-00597838

\section{HAL Id: hal-00597838 \\ https://hal.science/hal-00597838}

Submitted on 2 Jun 2011

HAL is a multi-disciplinary open access archive for the deposit and dissemination of scientific research documents, whether they are published or not. The documents may come from teaching and research institutions in France or abroad, or from public or private research centers.
L'archive ouverte pluridisciplinaire HAL, est destinée au dépôt et à la diffusion de documents scientifiques de niveau recherche, publiés ou non, émanant des établissements d'enseignement et de recherche français ou étrangers, des laboratoires publics ou privés. 
Diseases

Editorial Manager(tm) for European Journal of Clinical Microbiology \& Infectious

Manuscript Draft

Manuscript Number: EJCMID-D-10-00094R1

Title: Colonization pattern of coagulase-negative staphylococci in preterm neonates and the relation to bacteraemia.

Article Type: Article

Keywords: 1. Coagulase-negative Staphylococci, 2. Colonization, 3. Neonatal infections, 4. Preterm Newborns, 5. Typing methods

Corresponding Author: Dr. maria bjorkqvist, Ph.D.

Corresponding Author's Institution: Orebro University Hospital

First Author: maria bjorkqvist, M.D., Ph.D.

Order of Authors: maria bjorkqvist, M.D., Ph.D.; Magnus Liljedahl, M.D.; Johanna Zimmerman; Jens Schollin, M.D., Ph.D.; Bo Soderquist, M.D., Ph.D.

Abstract: Coagulase-negative staphylococci (CoNS) are the major cause of sepsis in extreme preterm (EPT) newborns, but data about the CoNS colonization in EPT newborns prior to invasive infection are limited.

Our aim was to describe the early establishment of the CoNS microflora in EPT newborns and to compare the colonization pattern in neonates with and without positive CoNS blood cultures.

From a cohort of 46 EPT neonates, newborns with positive CoNS blood culture were identified $(n=10)$ and compared with matched controls. Samples for bacterial cultures were obtained repetitively from nares, perineum, and umbilicus. All CoNS isolates were characterized using the PhenePlate system for biochemical fingerprinting.

Persistent CoNS strains were found on d 2-3 after delivery in 7/20 newborns, and there was a tendency for earlier colonization in nares than in perineum or umbilicus. The CoNS blood strains were prevalent in superficial sites prior to positive blood culture (11/14 blood strains), but no single invasive pathway was identified. Most CoNS blood strains (9/14) persisted on superficial sites after antibiotic treatment.

We hypothesize that the invasive pathways in neonatal CoNS sepsis are complex and that colonization of mucosal membranes and umbilical catheters might be of equal importance.

Response to Reviewers: The comments of the reviewer have been a great help for us when revising the manuscript, and we want to express our thanks to the reviewer for constructive criticism.

As listed below, we would like to respond to the reviewer's comments.

1. The reviewer asked wheather restriction of the cases to only those classified as having true sepsis (and thus exclusion of the cases most probably representing contaminants) would change the results. In the original version of the manuscript we decided to compare only "blood culture positive" vs "bloodculture negative" in order to avoid any kind of byas according to the classification in "sepsis" and "contaminants". However, we agree with the reviewer that comparison of "sepsis" - "non-sepsis" is 
of interest and has included that kind of analysis in the results (page 10 line 22 and page 10 line 48). In summary these comparisons show the same trends as for the whole study group, however the numbers of included sepsis-newborns are limited, and the differences thus not statistically significant. - Our proportion of newborns with true CoNS sepsis however $(6 / 46)$ however is similar to the incidence reported from other Western centra.

2. Why do we not present data on all 46 newborns? - In the present study we choose a case-control design and consider this as relevant, since only a minor proportion of the total cohort displayed a CoNS positive blood culture. However, we are just now analysing the data from all 46 preterms compared to healthy term newborns and plan to present these data in future manuscript.

3. Why do the findings of PFGE not quite match the PhP data? - A comment on this has been included in the Discusison (page 15 line 34).

4. Results of PFGE for typing validation of pairs of colonising and blood isolates identified as identical from $\mathrm{PhP}$ ? - data has been included in the results (page 11 line 56).

5. Missing reference in the discussion. - Error in the proof by us. - The last part of the sentence that would correspond to the missing reference was thought to be omitted already in the original manuscript. This is now corrected.

We hope that the reviewer will find the new version of the manuscript improved compared to the original version and suggest it for publication in EJCMID. 


\section{Colonization pattern of coagulase-negative staphylococci in}

\section{preterm neonates and the relation to bacteraemia}

Running title: Preterm CoNS colonization and bacteraemia

MARIA BJÖRKQVIST, ${ }^{1 *}$ MAGNUS LILJEDAHL, ${ }^{1}$ JOHANNA ZIMMERMANN, ${ }^{2}$ JENS SCHOLLIN, ${ }^{1}$ AND BO SÖDERQUIST ${ }^{2}$

Departments of ${ }^{1}$ Pediatrics and ${ }^{2}$ Clinical Microbiology, Örebro University Hospital, SE70185 Örebro, Sweden

"Corresponding author: Maria Björkqvist, Department of Pediatrics, Örebro University Hospital, SE-70185 Örebro, Sweden. Tfn + 4619 6021000, fax + 4619 187915, maria.bjorkqvist@orebroll.se

Financial support: Grants from the research committee of Örebro county, Sweden

Category of study: Clinical study

Word count of abstract: 198

Word count of manuscript: 4618 


\begin{abstract}
Coagulase-negative staphylococci (CoNS) are the major cause of sepsis in extreme preterm (EPT) newborns, but data about the CoNS colonization in EPT newborns prior to invasive infection are limited.

Our aim was to describe the early establishment of the CoNS microflora in EPT newborns and to compare the colonization pattern in neonates with and without positive CoNS blood cultures.
\end{abstract}

From a cohort of 46 EPT neonates, newborns with positive CoNS blood culture were identified $(n=10)$ and compared with matched controls. Samples for bacterial cultures were obtained repetitively from nares, perineum, and umbilicus. All CoNS isolates were characterized using the PhenePlate system for biochemical fingerprinting. Persistent CoNS strains were found on d 2-3 after delivery in 7/20 newborns, and there was a tendency for earlier colonization in nares than in perineum or umbilicus. The CoNS blood strains were prevalent in superficial sites prior to positive blood culture (11/14 blood strains), but no single invasive pathway was identified. Most CoNS blood strains (9/14) persisted on superficial sites after antibiotic treatment.

We hypothesize that the invasive pathways in neonatal CoNS sepsis are complex and that colonization of mucosal membranes and umbilical catheters might be of equal importance. 


\section{INTRODUCTION}

In healthy term infants and other non-compromised individuals, coagulase-negative staphylococci (CoNS) represent commensals with low invasive potential. However, in immunocompromised patients and those with infections related to indwelling medical devices, CoNS infections constitute a significant nosocomial problem. Preterm newborns, especially extreme preterm (EPT) infants, are prone to CoNS sepsis, since they are immunologically immature, have pervious skin and mucosal barriers, and are regularly exposed to invasive procedures during neonatal intensive care $(1,2)$. Although the acute mortality is low, CoNS sepsis is associated with a significant increase in acute neonatal morbidity, and most probably contributes to the development of post-inflammatory sequelae such as bronchopulmonary dysplasia and CP syndromes (3-5).

Neonatal CoNS infections represent late onset sepsis, with onset of symptoms at 7-15 days postnatal age (6). Since persistence of single CoNS clones for several years is well documented in neonatal intensive care units (NICUs) (7-10), as is the emergence of multi drug resistant CoNS strains (including glycopeptides) $(7,8)$, there is an urgent need for epidemiologic surveillance in the NICUs and preventive/infection control strategies against neonatal invasive CoNS infections $(11,8)$.

CoNS possess a unique ability to colonize the skin and mucous membranes, and newborn children become colonized by their first CoNS strains early after birth (12-14). In EPT newborns, most of these early colonizing CoNS strains are acquired from the NICU environment $(12,13)$. However, detailed data about the establishment of the CoNS microflora in EPT newborns, as well as the correlation between superficial and invasive isolates of CoNS, are limited. In order to elaborate better strategies for the prevention of neonatal CoNS septicaemia, further insights into the colonization pattern of CoNS in EPT newborns prior to deep infection are necessary. 
Methodological difficulties constitute the major reasons for our limited knowledge of the establishment and dynamics of the normal flora of the newborn. Pulsed field gel electrophoresis (PFGE) and other conventional genetic typing methods are laborious and not applicable to the large populations of bacterial isolates found in superficial samples, whereas earlier phenotypic typing methods do not show appropriate discriminatory power $(15,16)$. Phenotypical fingerprinting based on multiple biochemical characteristics might be a useful tool for comprehensive typing of larger numbers of CoNS isolates. One such biochemical fingerprinting assay is the PhenePlate system $(\mathrm{PhP})$, which is well validated as a screening tool for selection of related CoNS isolates (17).

The first aim of the present study was to describe the early establishment of the CoNS microflora in EPT newborns, and the second aim was to compare the colonization patterns in bacteraemic neonates with those in preterm infants without invasive CoNS infection. 


\section{MATERIAL AND METHODS}

\section{Patients}

Preterm newborns $<31 \mathrm{w}$ gestational age, born at the delivery ward at Örebro University hospital and admitted to the NICU between April 2004 and November 2006, were eligible for the study. Samples for bacterial cultures from nares, umbilicus, and perineum were obtained using sterile, dry cotton swabs at $24 \mathrm{~h}$ and on day 2 , d 5-6, d 7-8, and d 14 after delivery. Samples for blood cultures were initiated when clinically indicated. For sepsis evaluation, $0.5-1 \mathrm{ml}$ of blood was obtained by peripheral venous puncture or by a newly inserted vascular umbilical catheter.

During the study period, 46 newborns were consecutively enrolled. From this cohort, all newborns $\leq 28$ days of age with blood cultures yielding CoNS and no other pathogen were retrospectively identified and selected for further evaluation. For each case, a control newborn matched for birth date (first priority) and gestational age (second priority) and with blood cultures negative for CoNS was selected from the same original study population. Data concerning invasive infections and antibiotic treatment during the first $28 \mathrm{~d}$ of postnatal age were collected.

Neonatal sepsis was defined as a positive blood culture in combination with $\geq 3$ clinical signs consistent with infection and a peak C-reactive protein $\geq 20 \mathrm{mg} / \mathrm{L}$ within $72 \mathrm{~h}$ from onset of symptoms. Patients that were transferred to another hospital or died within $28 \mathrm{~d}$ of age were excluded.

The NICU at Örebro University Hospital provides full neonatal intensive care, except for major neonatal surgery. Immediate postnatal rescue measurements are performed with attention to sterile techniques. All NICU staff members are instructed to use $70 \%$ alcohol gel for hand disinfection and to wear plastic gloves when handling individual patients. 


\section{Bacterial samples}

Bacterial samples from superficial sites were cultured overnight on blood agar medium (4.25\% Columbia II Agar [BBL, Becton Dickinson, Baltimore, MD, USA], 0.3\% Agar No. 2 [LAB M Ltd., Bury, UK], and 5\% bovine blood). All the growth on the agar plates was sampled and dissolved in preservation medium (yeast extract; DIFCO Laboratories, Sparks, MD, USA) and horse serum added trypticase soy broth (TSB; BBL, Sparks MD, USA), and stored at $-70^{\circ} \mathrm{C}$ pending further analysis.

Samples for blood cultures were immediately administered into BACTEC Peds bottles (Becton Dickinson, Franklin Lakes, NJ, USA). A non-radiometric BACTEC 9240 system was used to detect bacteria and fungi.

Minimum inhibitory concentration (MIC) determinations were performed using the Etest (AB Biodisk, Solna, Sweden).

The species of the blood isolates of CoNS were identified biochemically by the ID32 Staph (bioMérieux, Marcy l'Etoile, France) and in some cases confirmed by sequencing the $r p o B$ gene, as previously described (18).

\section{PhenePlate system}

Bacterial samples from nares, umbilicus, and perineum were subcultured overnight at $37^{\circ} \mathrm{C}$ on blood agar medium. Ten CoNS colonies from each sample were randomly chosen and subsequently biochemically fingerprinted using the PhP system modified for typing of CoNS (PhPlate AB, Stockholm, Sweden), as previously described (17). In summary, the system consists of 96-well microtitre plates, each with four sets of 22 different reagents. Colour indicators visualize changes in $\mathrm{pH}$ as a result of bacterial metabolization of these substrates. The absorbance value for each reaction is measured repeatedly and the mean value is calculated for each reaction. The results for different strains are compared in pairs, and the 
correlation coefficients are clustered using the unweighted pair group method of arithmetic mean (UPGMA). In the present study the absorbance was measured after 16, 40, and 64 hours at $620 \mathrm{~nm}$ in accordance with the manufacturer's instructions, and isolates with a correlation coefficient $\geq 0.975$ were regarded as belonging to the same PhP type (19). All data processing, including optical readings and calculations of correlation coefficients as well as clustering dendrograms, was performed with PhenePlate software.

\section{$P F G E$}

Characterization of the CoNS isolates was performed by PFGE of chromosomal SmaI digests, prepared with the GenePath group I reagent kit (Bio-Rad Laboratories, Hercules, CA), and PFGE patterns were obtained with a contour-clamped homogenous electric field apparatus (GenePath System; Bio-Rad) as previously described (9). Differences in band patterns were processed and analyzed with Molecular Analyst Fingerprinting software (version 1.6; BioRad Laboratories, Hercules, CA, USA) using the Dice coefficient, and represented by unweighted pair grouping by mathematical averaging (UPGMA) with $1 \%$ band tolerance and $0.5 \%$ optimization, but finally documented by visual examination and interpreted as recommended by Tenover et al. (20).

\section{Ethics}

The regional committee for medical research ethics approved the study, and parental informed consent was required for participation. 


\section{Statistics}

The Mann-Whitney U test and Student's unpaired $t$ test were used for two-group assessments of differences in continuous variables. Fisher's exact test was used to estimate differences in proportions.

The phenotypic diversity of the CoNS isolates was calculated as Simpson's Diversity Index $\left(D_{i}\right)$ using the PhenePlate software. A high $D_{i}$ indicates that the analyzed isolates are evenly distributed among the different bacterial phenotypes. The $\mathrm{D}_{\mathrm{i}}$ was calculated both for all isolates obtained from each patient and for selected isolates; specifically, only one of each set of identical isolates (with a correlation coefficient $\geq 0.975$ ) found at each sampling site and point of time was included. 


\section{RESULTS}

\section{Patients}

Ten preterm newborns with blood cultures positive for CoNS (patients A-J), representing 11 episodes, were identified during the study period, and they were matched with 10 controls. Two pairs of cases and controls were twins. The demographic characteristics are given in Table 1. One patient (patient G) contributed with two episodes. Six of the eleven episodes met the criteria for CoNS sepsis.

One of the controls was diagnosed with E. coli bacteraemia at delivery. No positive blood cultures were found among the other nine controls.

All 10 blood culture-positive newborns and 6 of the controls received parenteral antibiotics (Table 1). Cefotaxime + gentamicin was administered to 14 newborns (10 cases and 4 controls) at one or more episodes. Vancomycin was administered to 8 of the 9 cases with meticillin-resistant CoNS isolates in blood cultures (Fig. 1) and to one of the controls. All newborns had an umbilical arterial or venous catheter inserted soon after birth, and in 4 of the 10 blood culture-positive patients the umbilical catheter was still present at the time of a positive CoNS blood culture. The duration of indwelling umbilical catheters did not differ between the patients and the controls (median $5 \mathrm{~d}$, range $2 \mathrm{~d}-8 \mathrm{~d}$ ).

\section{Consecutive CoNS colonization by location in cases and controls}

Seven of the 20 investigated preterm newborns were already colonized by $1-6$ different CoNS isolates at $<24 \mathrm{~h}$ after delivery, one newborn in the umbilicus and the others in the nares or perineum. Except in one newborn, none of these initially recovered CoNS strains persisted beyond $24 \mathrm{~h}$ of age. Soon thereafter, however, a more persistent CoNS microflora was established. Fourteen of the 18 newborns that were sampled on d 2-3 were colonized in the 
nares $(n=10)$ and/or in the umbilicus $(n=5)$ or perineum $(n=7)$, and in 13 of these 18

newborns one or more of these strains could still be recovered on d 14 . On d 5-7 most newborns were colonized both in the nares (19/20) and in the umbilicus (17/20). The numbers of isolates eligible for further investigation varied among the included newborns, since some cultures from superficial sites (especially those sampled at the first event) yielded fewer than the 10 colonies of CoNS per sample that were stipulated for $\mathrm{PhP}$, and some displayed no growth of bacteria at all. From the blood culture-positive group of newborns, a median of 105 isolates per case (range 62-151) were eligible for further investigation, compared with a median of 85 isolates in the controls (range 6-110, $\mathrm{p}=0.034$ ). The same tendency, though not statistically significant, was found when the comparison was restricted to the cases classified as representing true sepsis $(n=6)$ and their corresponding controls; From the sepsis cases a median of 101 isolates per case were retrieved (range 62 100) compared to a median of 85 isolates from the sepsis controls $(n=6$, range $75-110, p=$ 0.34). In total, 1916 CoNS isolates were characterized by $\mathrm{PhP}$.

\section{Diversity in CoNS colonization in cases and controls}

A representative clustering dendrogram based on the $\mathrm{PhP}$ characteristics of all CoNS isolates retrieved from one individual is depicted in Fig. 2. The $\mathrm{D}_{\mathrm{i}}$ for the cases (mean 0.88, SD 0.062) was significantly higher than for the controls (mean $0.79, \mathrm{SD} 0.012, \mathrm{p}=0.04$ ) when all isolates for each individual newborn were included in the analysis ( $\mathrm{n}=1896$ isolates). Also the $\mathrm{D}_{1}$ for group of cases representing sepsis (mean $0.88, \mathrm{SD} 0.047, \mathrm{n}=6$ ) tended to be higher compared to the sepsis controls (mean 0.78, SD 0.129), however that difference was not statistically significant $(\mathrm{p}=0.125)$. When only one isolate representing the same site and the same point of time for isolation within the same phenotypic cluster was selected for further assessment ( $\mathrm{n}=674$ isolates in 20 infants), there were no significant differences in $\mathrm{D}_{\mathrm{i}}$ or in 
numbers of phenotypic clusters and single isolates between the two groups of newborns (Table 2).

\section{Blood cultures}

Fifteen blood isolates of CoNS representing 11 episodes were recovered from patients A-J. Four patients each contributed two isolates from the same blood culture. One sepsis isolate (patient $\mathrm{G}$, episode 1) could not be recultivated. Of the remaining 14 CoNS isolates, 11 were identified as S. epidermidis, one as S. lugdunensis, and two as S. capitis. All but two of the blood isolates were meticillin-resistant, and all isolates were sensitive to vancomycin (Table $3)$.

The blood isolates were compared according to phenotype $(\mathrm{PhP})$ and also characterized genotypically (PFGE), as illustrated in Fig. 3. Some differences were found between the phenotypic and genotypic results, reflecting the different properties of the two methods. The

majority of the $S$. epidermidis strains appeared in two clusters phenotypically, though with a correlation coefficient not reaching $\geq 0.975$. Three pairs of CoNS strains were genetically closely related according to the PFGE patterns. The species S. lugdunensis and S. capitis differed both phenotypically and genotypically from the S. epidermidis (Fig. 3).

\section{Superficial colonization of CoNS in relation to invasiveness and antibiotic treatment}

The invasive blood isolates were prevalent among the colonizing flora in the neonates prior to positive blood cultures. In 11/14 of the blood isolates, skin or mucosal isolates belonging to the same phenotypic cluster were also found on superficial sites of the same patient, and the colonization rate did not differ between nares ( $n=6$ strains), perineum ( $n=6$ strains), and umbilicus ( $n=4$ strains). In addition, the PFGE patterns were indistinguishable in all but one of the cases when comparing the superficial isolates with blood isolates that clustered 
together, Three blood isolates did not cluster with any colonizing isolates obtained from their corresponding patients and thus were regarded as single isolates. Two of these isolates (patients B1: S. lugdunensis and J1: S. epidermidis) originated from blood cultures yielding two strains of CoNS of different species, and one isolate (patient F) originated from a blood culture obtained at $24 \mathrm{~h}$ after delivery. The colonization patterns of the individual patients in relation to positive blood culture and antibiotic exposure are shown in Fig. 1.

In the majority of the patients, isolates belonging to the same phenotypic cluster as the blood isolates still persisted on superficial sites after antibiotic treatment (9/14 isolates, Fig. 1). Some of the CoNS blood strains were also prevalent among the superficial CoNS microflora in the blood culture-negative controls. For example, superficial isolates clustering together with the blood isolates 06B-861, 06B-105, and 06B-080 were found in at least half of the control patients.

\section{CoNS colonization following Caesarean section or vaginal delivery}

Delivery mode was not associated with any differences in CoNS colonization. Seventy percent of the included newborns were delivered by acute Caesarean section (CS); of these, 7/14 infants were colonized by CoNS at $24 \mathrm{~h}$ postnatal age compared to $1 / 6$ of the vaginally delivered newborns $(\mathrm{p}=0.32)$. CoNS strains that still persisted on $\mathrm{d} 14$ were recovered on day $2-3$ in $9 / 12$ newborns delivered by $\mathrm{CS}$ and in $4 / 6$ delivered vaginally $(p=0.99)$. Six newborns (5 in the CS group and one vaginally delivered) were born after preterm rupture of the membranes. The CoNS colonization patterns in these six newborns were similar to thosein all the other newborns (data not shown). 


\section{DISCUSSION}

In the present study, we found phenotyping by biochemical fingerprinting $(\mathrm{PhP})$ to be a useful tool for screening of related isolates among a vast number of CoNS isolates. This made it possible to investigate the establishment of the colonizing CoNS microflora in EPT newborns with a higher discriminatory power than most earlier methods could offer $(15,16)$. In two-thirds of the investigated preterm newborns, colonizing CoNS strains that persisted during the first postnatal weeks were already retrieved on postnatal day $2-3$. These findings are in contrast to earlier reports in which the early CoNS microflora was found to be transient and characterized by a considerable day-to-day variation $(12-14,21)$. However, data regarding CoNS colonization in preterm newborns are limited, and most studies were conducted in the early 1990s. Since then, neonatal intensive care has dramatically changed; early enteral feeding, decreased incidence of severe respiratory distress, and consistent education of all NICU staff members in hand disinfection are examples of achievements that most probably affect the CoNS microflora in NICU treated newborns nowadays. In comparison to infants with negative CoNS cultures, blood culture-positive newborns showed a higher number of CoNS isolates on colonizing sites as well as a tendency to be colonized earlier after delivery. This might reflect increased transmission of CoNS strains from NICU staff members as a result of more frequent and more acute handling of the sicker newborns, who are at greater risk for later acquiring CoNS bacteraemia. The majority of the blood strains were prevalent in the superficial microflora on several sites before being retrieved from the blood, but no single predominating invasive pathway could be identified. This indicates that several loci may serve as the entry in cases of neonatal CoNS bacteraemia. We found indications for earlier CoNS colonization in the nares than in the perineum or in the umbilicus, and $80 \%$ of the cases were colonized in the nares prior to, or at the same time as, 
acquisition of positive CoNS blood cultures. Relatively stable CoNS reservoirs in the nares

and faeces of preterm newborns have been reported previously (13). In adult immunocompromised patients, the possibility of mucosal colonization as an entry for invasive CoNS infections has been reviewed by Costal et al. (22). Mucosal barriers in preterm newborns are generally penetrable, but the epithelium of the upper respiratory tract is, in addition, regularly traumatized by the insertion of gastric sounds, CPAP adapters, air jet beams, and nasotracheal tubes, as well as by airway suction procedures. The numbers of patients in the present study are too small to draw any firm conclusions, but it may be worth considering that CoNS bacteraemia in newborns might be associated with various invasive entries.

Most investigations of pathogenic mechanisms in CoNS infections so far have focused on bacterial interactions with fibrinogen-coated polystyrene surfaces, for example, the bacterial formation of biofilm or expression of adhesion molecules, as well as other factors of importance in infections associated with indwelling medical devices $(23,24)$. These pathogenic mechanisms are probably important in the pathogenesis of neonatal infections, but no specific virulence factors that discriminate between invasive and colonizing neonatal CoNS isolates have been identified $(7,25,26)$. In EPT newborns, bacterial interactions with skin and mucosal membranes might be as important as colonization of umbilical catheters, and hence a broad approach is necessary when investigating the pathogenesis in neonatal CoNS infections.

We did not find any differences in CoNS colonization patterns associated with delivery mode or preterm rupture of the membranes, and thus no indications of maternal transferral of persisting or invasive CoNS strains, which is in accordance with earlier reports $(12,13)$. It is well documented that EPT newborns in the NICU environment are predominantly colonized by nosocomial strains of CoNS, and that these strains may persist in the NICU for years, 
which emphasizes the concern of nosocomial spread of multiresistant bacterial strains in the

In one of the patients, the blood culture yielded both a strain of S. epidermidis and an isolate of S. lugdunensis. Except for one case report (27), S. lugdunensis is not known to cause neonatal bacteraemia, though this species appears to be more virulent than most other CoNS species (28). It was not possible to determine if this finding of S. lugdunensis represents true sepsis or a contaminant transferred by the NICU staff. However, S. lugdunensis was not found in any of the superficial skin cultures from any of the newborns.

Since $\mathrm{PhP}$ reveals phenotypic and PFGE genotypic polymorphism, it is not reasonable to expect a $100 \%$ concordance between these two typing methods. In an earlier study, we evaluated $\mathrm{PhP}$ for long-term characterization of CoNS blood isolate, and found the method less discriminatory than PFGE (9). In the present study, multiple CoNS isolates that were retrieved from each single individual during a limited time period were compared. For this type of investigation, $\mathrm{PhP}$ has shown a satisfactory capacity for identification of phenotypically related CoNS isolates (17), but for long-term surveillance, robust genetic typing methods that could be carried out on large bacterial populations are warranted. In summary, we report early acquisition of nosocomial CoNS strains in the colonizing microflora of preterm newborns, and note that these bacterial strains are able to persist regardless of antibiotic treatment. The invasive pathways in neonatal CoNS bacteraemia are 
probably complex, and this should be taken into consideration in studies both of bacterial virulence factors and of when to carry out preventive strategies against invasive CoNS infections in preterm newborns.

\section{ACKNOWLEDGMENTS}

The authors wish to thank Bengt Hellmark for the $r p o B$ sequencing. The study was supported by grants from the research committee of Örebro county. 


\section{REFERENCES}

1. Stoll BJ, Hansen N, Fanaroff AA, Wright LL, Carlo WA, Ehrenkranz RA, Lemons JA, Donovan EF, Stark AR, Tyson JE, Oh W, Bauer CR, Korones SB, Shankaran S, Laptook AR, Stevenson DK, Papile LA, Poole WK (2002). Late-onset sepsis in very low birth weight neonates: the experience of the NICHD Neonatal Research Network. Pediatrics 110:285-291

2. Fanaroff AA, Stoll BJ, Wright LL, Carlo WA, Ehrenkrans RA, Stark AR, Bauer CR, Donovan EF, Korones SB, Laptook AR, Lemons JA, Oh W, Papile LA, Shankaran S, Stevenson DK, Tyson JE, Poole WK; NICHD Neonatal Research Network (2007). Trends in neonatal morbidity and mortality for very low birthweight infants. Am J Obstet Gynecol 196:147.e1-8

3. Liljedahl M, Bodin L, Schollin J (2004). Coagulase-negative staphylococcal sepsis as a predictor of bronchopulmonary dysplasia. Acta Paediatr 93:211-215

4. Speer CP (2003). Inflammation and bronchopulmonary dysplasia. Semin Neonatol $8: 29-38$

5. Wilson-Costello D, Borawski E, Friedman MA, Redline R, Fanaroff AA, Hack M (1998). Perinatal correlates of cerebral palsy and other neurologic impairment among very low birth weight children. Pediatrics 102:315-322

6. Isaacs D (2003). A ten year, multicentre study of coagulase negative staphylococcal infections in Australian neonatal units. Arch Dis Child Fetal Neonatal Ed 88:F89F93

7. Klingenberg C, Rønnestad A, Anderson AS, Abrahamsen TG, Zorman J, Villaruz A, Flægstad T, Otto M, Eriscon Sollid JE (2007). Persistent strains of coagulasenegative staphylococci in a neonatal intensive care unit: virulence factors and invasiveness. Clin Microbiol Infect 13:1100-1111 
8. Villari P, Saturno C, Iacuzio L (2000). Molecular epidemiology of Staphylococcus epidermidis in a neonatal intensive care unit over a three-year period. J Clin Microbiol 38:1740-1746

9. Björkqvist M, Söderquist B, Törnqvist E, Sjöberg L, Fredlund H, Kühn I, ColqueNavarro P, Schollin J (2002). Phenotypic and genotypic characterisation of blood isolates of coagulase-negative staphylococci in the newborn. APMIS 110:332-339

10. Vermont CL, Hartwig NG, Fleer A, de Man P, Verbrugh H, van den Anker J, de Groot R, van Belkum A (1998). Persistence of clones of coagulase-negative staphylococci among premature neonates in neonatal intensive care units: two-center study of bacterial genotyping and patient risk factors. J Clin Microbiol 36:24852490

11. Center KJ, Reboli AC, Hubler R, Rodgers GL, Long SS (2003). Decreased vancomycin susceptibility of coagulase-negative staphylococci in a neonatal intensive care unit: evidence of spread of Staphylococcus warneri. J Clin Microbiol 41:4660-4665

12. Keyworth N, Millar MR, Holland KT (1992). Development of cutaneous microflora in premature neonates. Arch Dis Child 67:797-801

13. Eastick K, Leeming JP, Bennett D, Millar MR (1996). Reservoirs of coagulase negative staphylococci in preterm infants. Arch Dis Child Fetal Neonatal Ed 74:F99-104

14. D’Angio CT, McGowan KL, Baumgart S, St Geme J, Harris MC (1989). Surface colonization with coagulase-negative staphylococci in premature neonates. J Pediatr 114:1029-1034

15. Sloos JH, Dijkshoorn L, Vogel L, van Boven CPA (2000). Performance of phenotypic and genotypic methods to determine the clinical relevance of serial blood 
isolates of staphylococcus epidermidis in patients with septicemia. J Clin Microbiol $38: 2488-2493$

16. Spare MK, Tebbs SE, Lang S, Lambert PA, Worthington T, Lipkin GW, Elliott TS (2003). Genotypic and phenotypic properties of coagulase-negative staphylococci causing dialysis catheter-related sepsis. J Hosp Infect 54:272-278

17. Nilsdotter-Augustinsson $\AA$, Koskela A, Öhman L, Söderquist B (2007). Characterization of coagulase-negative staphylococci isolated from patients with hip prostheses: use of phenotypic and genotypic analyses, including tests for the presence of the ica operon. Eur J Clin Microbiol Infect Dis 26:255-265

18. Hellmark B, Söderquist B, Unemo M (2009). Simultaneous species identification and detection of rifampicin resistance in staphylococci by sequencing of the rpoB gene. Eur J Clin Microbiol Infect Dis 28:183-190

19. Jung K, Brauner A, Kühn I. Typing of Coagulase-negative staphylococci from peritonitis in CAPD-patients by the PhP-CS system and REA (1995). APMIS 103:679-685

20. Tenover FC, Arbeit RD, Goering RV, Mickelsen PA, Murray BE, Persing DH, Swaminathan B (1995). Interpreting chromosomal DNA restriction patterns produced by pulsed-field gel electrophoresis: criteria for bacterial strain typing. J Clin Microbiol 33:2233-2239

21. Hall SL, Riddell SW, Barnes WG, Meng L, Hall RT (1990). Evaluation of coagulase-negative staphylococcal isolates from serial nasopharyngeal cultures of premature infants. Diagn Microbiol Infect Dis 13:17-23

22. Costa SF, Miceli MH, Anaissie EJ (2004). Mucosa or skin as source of coagulasenegative staphylococcal bacteraemia? Lancet Infect Dis 4:278-286 
23. Vuong C, Otto M (2002). Staphylococcus epidermidis infections. Microbes Infect 4:481-489.

24. von Eiff C, Peters G, Heilmann C (2002). Pathogenesis of infections due to coagulase-negative staphylococci. Lancet Infect Dis 2:677-685

25. de Silva GD, Kantzanou M, Justice A, Massey RC, Wilkinson AR, Day NP, Peacock SJ (2002). The ica operon and biofilm production in coagulase-negative Staphylococci associated with carriage and disease in a neonatal intensive care unit. J Clin Microbiol 40:382-388.

26. Bradford R, Abdul Manan R, Daley AJ, Pearce C, Ramalingam A, D’Mello D, Mueller Y, Uahwatanasakul W, Qu Y, Grando D, Garland S, Deighton M (2006). Coagulase-negative staphylococci in very-low-birth-weight infants: inability of genetic markers to distinguish invasive strains from blood culture contaminants. Eur J Clin Microbiol Infect Dis 25:283-290

27. Tee WSN, Soh SY, Lin R, Loo LH (2003). Staphylococcus lugdunensis carrying the mecA gene causes catheter-associated bloodstream infection in premature neonate. $\mathbf{J}$ Clin Microbiol 41:519-520

28. Frank KL, del Pozo JL, Patel R (2008). From clinical microbiology to infection pathogenesis: how daring to be different works for Staphylococcus lugdunensis. Clin Microbiol Rev 21:111-133 
ABBREVIATIONS

CoNS $=$ Coagulase-negative staphylococci

$\mathrm{EPT}=$ extreme preterm

PFGE $=$ pulsed field gel electrophoresis

$\mathrm{PhP}=$ PhenePlate system

$\mathrm{MIC}=$ minimum inhibitory concentration

UPGMA = unweighted pair grouping by mathematical averaging

$\mathrm{D}_{\mathrm{i}}=$ diversity index 
Table 1. Preterm newborns with blood cultures yielding CoNS (cases) and blood culturenegative controls.

$\begin{array}{ll}\text { Cases }(\mathrm{n}=10) & \text { Controls }(\mathrm{n}=10) \\ \text { Median (range) } & \text { Median (range) }\end{array}$

\begin{tabular}{llll}
\hline Gestational age & $29 \mathrm{w}+3 \mathrm{~d}$ & $29 \mathrm{w}+3 \mathrm{~d}$ & 0.88 \\
& $(24 \mathrm{w}+3 \mathrm{~d}-30 \mathrm{w}+3 \mathrm{~d})$ & $(24 \mathrm{w}+1 \mathrm{~d}-30 \mathrm{w}+4 \mathrm{~d})$ & n.s. \\
Birth weight & $1067 \mathrm{~g}$ & $1282 \mathrm{~g}$ & 0.66 \\
& $(590 \mathrm{~g}-1495 \mathrm{~g})$ & $(600 \mathrm{~g}-1570 \mathrm{~g})$ & n.s. \\
& $3.5 \mathrm{~d}$ & $1.0 \mathrm{~d}$ & 0.16 \\
Postnatal age at start of first & $(0 \mathrm{~d}-10 \mathrm{~d})$ & $(0 \mathrm{~d}-5 \mathrm{~d})$ & n.s. \\
antibiotic treatment & $9.0 \mathrm{~d}$ & $4.5 \mathrm{~d}$ & 0.10 \\
Duration of antibiotic treatment & $(4 \mathrm{~d}-14 \mathrm{~d})$ & $(0 \mathrm{~d}-13 \mathrm{~d})$ & n.s. \\
$<14 \mathrm{~d}$ after delivery & $10 \mathrm{~d}$ & $4.5 \mathrm{~d}$ & 0.02 \\
Duration of antibiotic treatment & $(6 \mathrm{~d}-19 \mathrm{~d})$ & $(0 \mathrm{~d}-23 \mathrm{~d})$ & \\
$\leq 28 \mathrm{~d}$ after delivery & & & \\
\hline
\end{tabular}

*Mann-Whitney U-test

n.s. $=$ not significant 
Table 2. Diversity in colonizing CoNS isolates from nares, perineum, and umbilicus in preterm newborns with positive CoNS blood culture and in controls. In cases of multiplicates within an individual phenotypic cluster of isolates representing the same locus and point of time for isolation, only one of these isolates is selected for the analysis.

\begin{tabular}{llll}
\hline & $\begin{array}{l}\text { Cases } \mathrm{n}=10 \\
\text { mean (SD) }\end{array}$ & $\begin{array}{l}\text { Controls } \mathrm{n}=10 \\
\text { mean (SD) }\end{array}$ & $\mathrm{p}^{\S}$ \\
\hline Numbers of isolates included & $37.0(11.40)$ & $30.6(8.35)$ & 0.16 \\
Diversity index & $0.907(0.033)$ & $0.902(0.037)$ & 0.739 \\
Numbers of PhP clusters & $6.7(2.16)$ & $5.8(2.70)$ & 0.42 \\
Numbers of single isolates & $10.8(3.39)$ & $9.0(4.19)$ & 0.30 \\
\hline
\end{tabular}

$\S$ unpaired $t$ test 
Table 3. CoNS blood isolates from preterm newborns. $\mathrm{S}=$ sensitive and $\mathrm{R}=$ resistant

\begin{tabular}{|c|c|c|c|c|c|}
\hline \multirow[t]{2}{*}{ Patient } & \multirow[t]{2}{*}{ Strain } & \multirow[t]{2}{*}{ Species } & \multicolumn{3}{|c|}{ Antibiotic resistance } \\
\hline & & & Meticillin & Gentamicin & Vancomycin \\
\hline A & 04B475 & S. epi & $\mathrm{R}$ & $\mathrm{R}$ & S \\
\hline \multirow[t]{2}{*}{$\bullet B$} & 06B860 & S. lugdunensis & $\mathrm{S}$ & $\mathrm{S}$ & $\mathrm{S}$ \\
\hline & 06B861 & S. epi & $\mathrm{R}$ & $\mathrm{S}$ & $\mathrm{S}$ \\
\hline \multirow[t]{2}{*}{$\mathrm{C}$} & 05B780G & S. epi & $\mathrm{R}$ & $\mathrm{R}$ & $\mathrm{S}$ \\
\hline & 05B 780V & S. capitis & $\mathrm{R}$ & $\mathrm{R}$ & $\mathrm{S}$ \\
\hline$\bullet \mathrm{D}$ & 06B195 & S. epi & $\mathrm{R}$ & $\mathrm{S}$ & $\mathrm{S}$ \\
\hline$\bullet \mathrm{E}$ & 06B 105 & S. epi & $\mathrm{S}$ & $\mathrm{S}$ & $\mathrm{S}$ \\
\hline $\mathrm{F}$ & 04B360 & S. epi & $\mathrm{R}$ & $\mathrm{S}$ & $\mathrm{S}$ \\
\hline $\mathrm{G}^{*}$ & 04B671 & S. epi & $\mathrm{R}$ & $\mathrm{R}$ & $\mathrm{S}$ \\
\hline \multirow[t]{2}{*}{$\bullet \mathrm{H}$} & 06B081 & S. epi & $\mathrm{R}$ & $S$ & $S$ \\
\hline & 06B080 & S. epi & $\mathrm{R}$ & $\mathrm{S}$ & $\mathrm{S}$ \\
\hline$\bullet I$ & 06B558 & S. epi & $\mathrm{R}$ & $\mathrm{S}$ & $\mathrm{S}$ \\
\hline \multirow[t]{2}{*}{$\mathrm{J}$} & $06 \mathrm{~B} 709$ & S. epi & $\mathrm{R}$ & $\mathrm{R}$ & $\mathrm{S}$ \\
\hline & $06 \mathrm{~B} 716$ & S. capitis & $\mathrm{R}$ & $\mathrm{R}$ & $\mathrm{S}$ \\
\hline
\end{tabular}

Minimum inhibitory concentration (MIC) cut-off values for the bacterial isolates to be classified as sensitive to the different antibiotics: Meticillin MIC <1 mg/L; Gentamicin MIC $<0.25 \mathrm{mg} / \mathrm{L}$; Vancomycin MIC <4 mg/L; $\bullet$ indicates a sepsis patient; $S$. epi $=$ S. epidermidis * second episode 


\section{FIGURE LEGENDS}

Figure 1. Relation between blood isolates of CoNS, superficial colonization by the same bacterial strain, and antibiotic treatment in CoNS blood culture-positive preterm newborns $(\mathrm{n}=10) . \quad \bullet=$ sepsis patient $\mathbf{U}=$ umbilicus, $\mathbf{P}=$ perineum and $\mathbf{N}=$ nares. $n . d=$ superficial bacterial culture not obtained.

$\uparrow$ indicates the occurrence of positive CoNS blood culture $(\uparrow)=$ blood isolate not recultivated.

Bars indicate antibiotic treatment: = = bensylpenicillin + garamicin, WIII = cefotaxim + garamicin, and $=$ vancomycin.

Figure 2. A representative clustering dendrogram based on the $\mathrm{PhP}$ characteristics of all CoNS isolates retrieved from one individual preterm newborn. $\mathrm{N}=$ nares and $\mathrm{P}=$ perineum. No positive cultures from the umbilicus were obtained in this patient.

\section{Figure 3.}

Phenotypic and genotypic characteristics of CoNS blood isolates $(n=14)$ from preterm newborns $(\mathrm{n}=10)$. The upper clustering dendrogram describes the phenotypic relationship between the bacterial isolates based on the $\mathrm{PhP}$ characteristics. The lower dendrogram describes the genotypic characteristics of the same isolates based on PFGE as visualized on the corresponding agarose gel. 
Figure 1

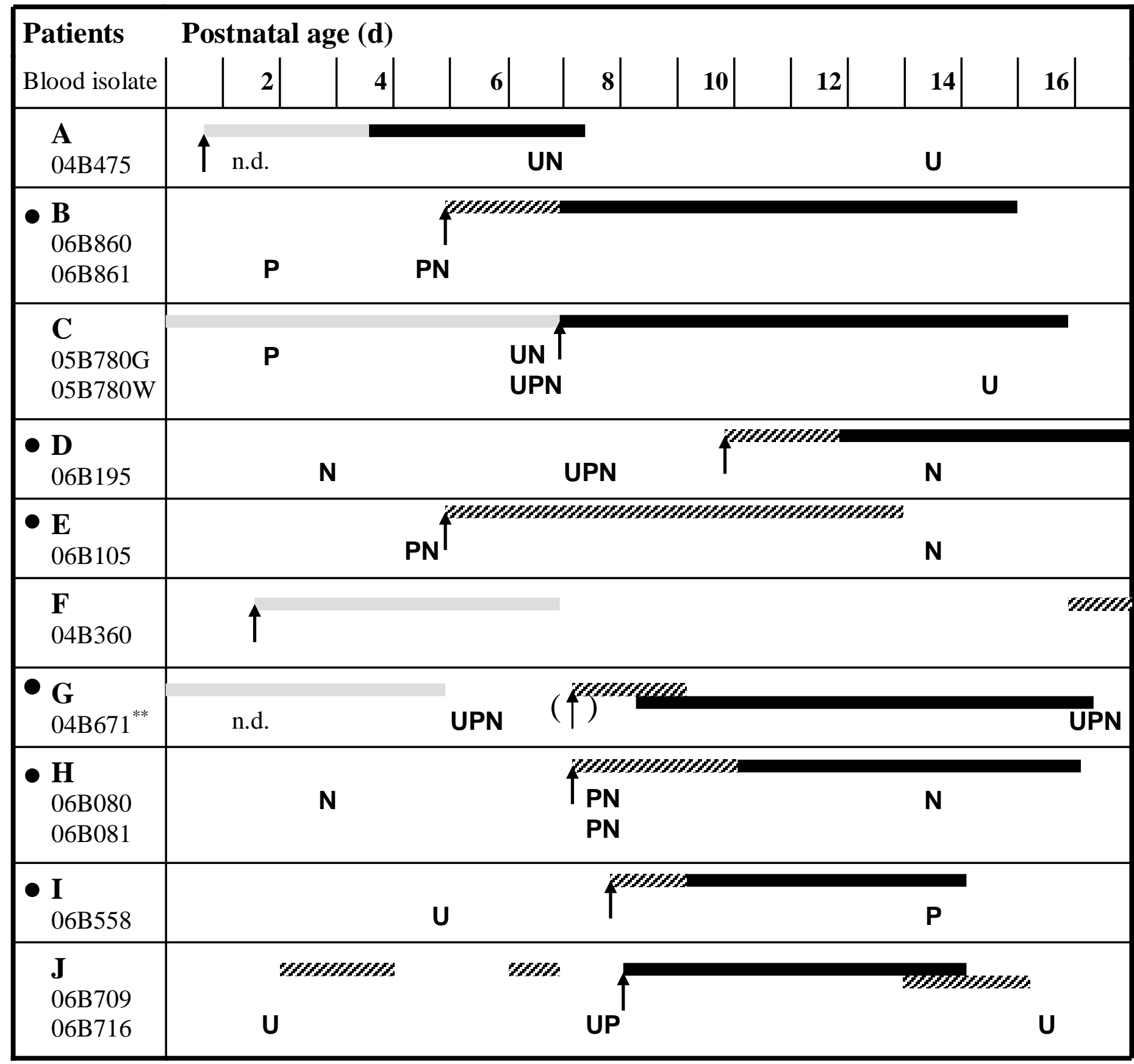

** Retrieved on d 23 
Figure 2

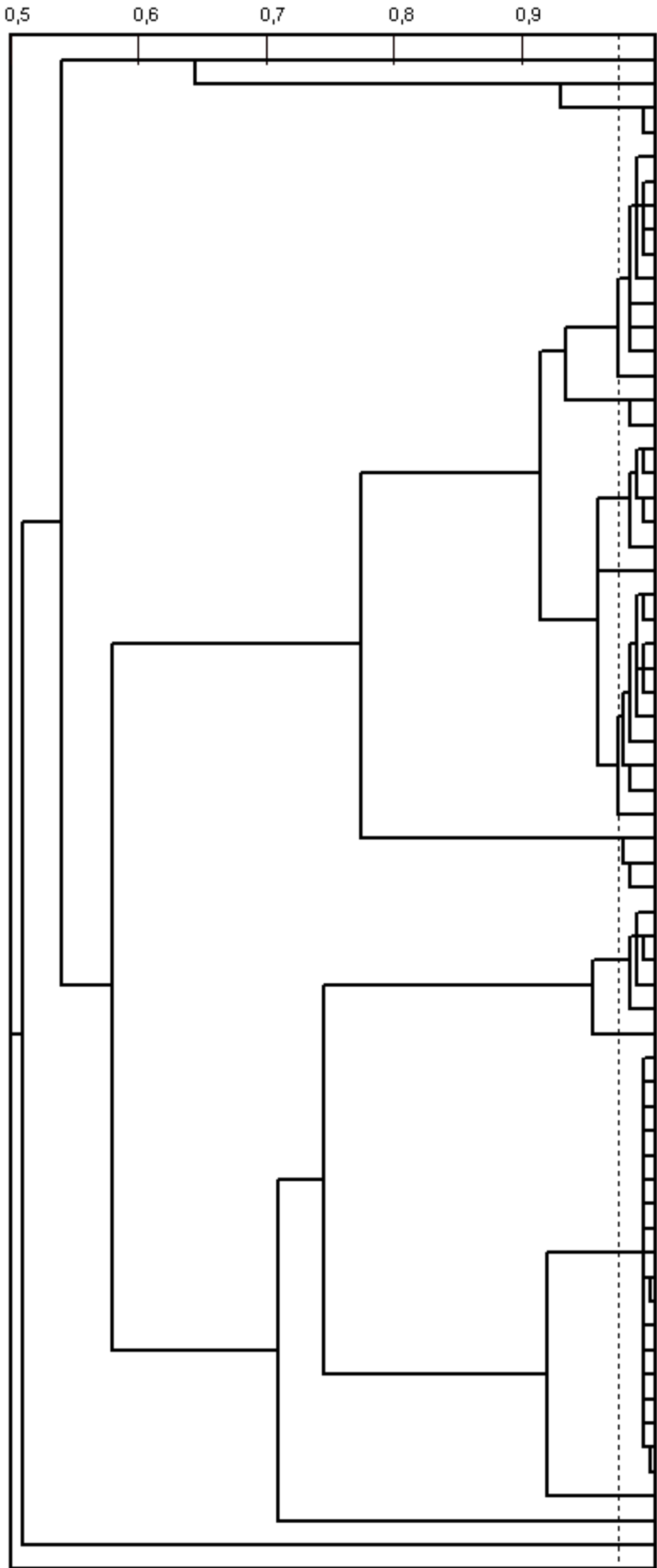

06日-860 (Blood) B6-2 ( $P$ d15) B6-6 ( $P$ d15) B6-10 ( $\mathrm{P}$ d15) 068-861 (Blood) B1 4 ( $\mathrm{P} \mathrm{d} 2$ ) $B 29$ (Nd5) B2 8 (N d5) B1 $9(\mathrm{P} \mathrm{d} 2)$ $\mathrm{B} 27$ (N d5) B4-3 (N 7d) B4-6 (N 7d) B3 4 ( $\mathrm{C}$ d5) B4-2 (N 7d) B1 $3(\mathrm{P} \mathrm{d} 2)$ B1 $6(\mathrm{P} \mathrm{d} 2)$ B1 $2(\mathrm{P} \mathrm{d} 2)$ B2 6 (N d5) B1 5 ( $\mathrm{P} \mathrm{d}$ 2) B5-5 ( $\mathrm{P}$ d7) B2 3 (N d5) $\mathrm{B} 310$ ( $\mathrm{P}$ d5) B1 8 ( $\mathrm{P} \mathrm{d} 2)$ B4-7 (N 7d) B4-4 (N 7d) B4-5 (N 7d) B4-8 (N 7d) B4-10 (N 7d) B3 9 ( $P$ d5) B3 8 ( $P$ d5) B4-9 (N 7d) B3 7 ( $\mathrm{P}$ d5) B5-3 ( $\mathrm{P} \mathrm{d7})$ 66-3 ( $P$ d15) 86-9 ( $P$ d15) B1 $1(\mathrm{P} \mathrm{d} 2)$ B 31 ( $P$ d5) B3 5 ( $\mathrm{P}$ d5) B3 6 ( $\mathrm{P}$ d5) B3 $3(P d 5)$ B3 2 ( $\mathrm{P}$ d5) B1 10 (P d2) B2 2 ( $(\mathrm{N} d 5$ ) $\mathrm{B} 24$ (N d5) B2 5 (N d5) B5-6 ( $\mathrm{P}$ d7) B2 10 (N d5) B5-4 ( $\mathrm{P} \mathrm{d7}$ ) B5-2 ( $\mathrm{P} \mathrm{d7}$ ) B5-1 ( $\mathrm{P} \mathrm{d} 7$ ) B5-7 ( $\mathrm{P} \mathrm{d7})$ B6-4 (P d15) 65-8 ( $\mathrm{P} \mathrm{d7}$ ) B5-9 ( $\mathrm{P} \mathrm{d7})$ B5-10 ( $\mathrm{P} \mathrm{d} 7)$ 86-1 ( $\mathrm{P}$ d15) 86-8 ( $\mathrm{P}$ d15) 86-5 (P d15) 66-7 ( $\mathrm{P}$ d15) B2 1 (N d5) B1 $7(\mathrm{P} \mathrm{d} 2)$ B4-1 (N 7d) 
Figure 3

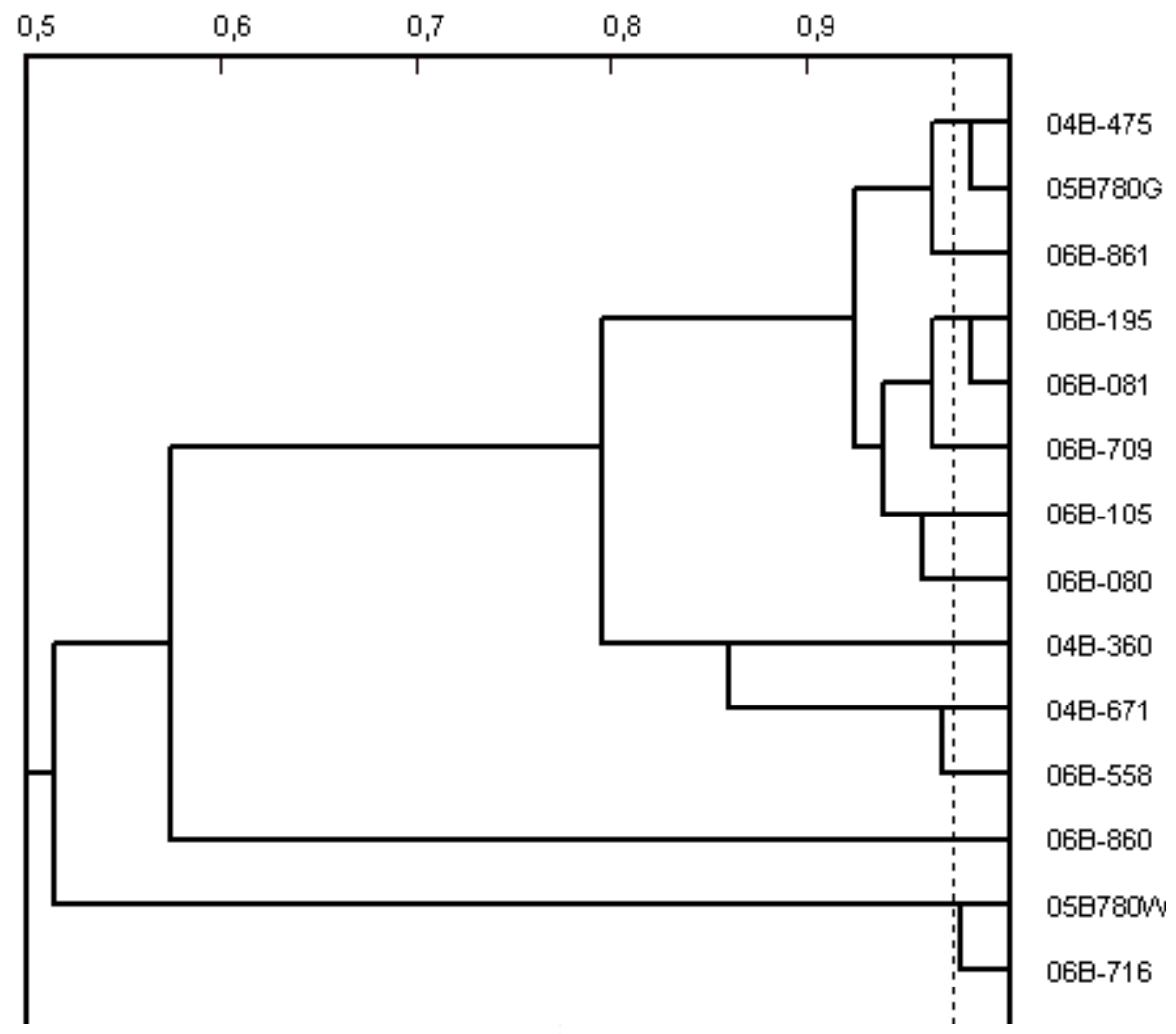

$\begin{array}{lllllll}40 & 50 & 60 & 70 & 80 & 90 & 100\end{array}$
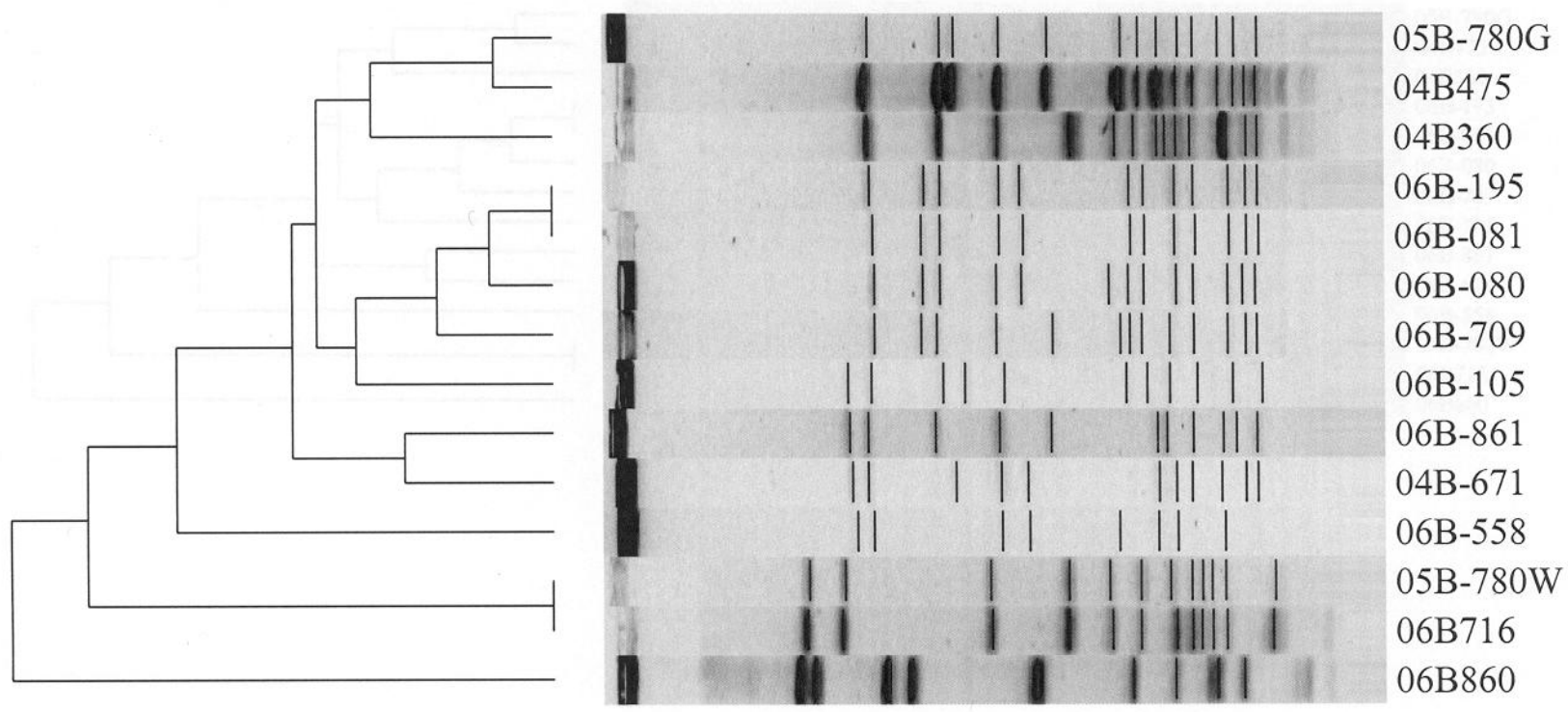
The comments of the reviewer have been a great help for us when revising the manuscript, and we want to express our thanks to the reviewer for constructive criticism.

As listed below, we would like to respond to the reviewer's comments.

1. The reviewer asked wheather restriction of the cases to only those classified as having true sepsis (and thus exclusion of the cases most probably representing contaminants) would change the results. - In the original version of the manuscript we decided to compare only "blood culture positive" vs "bloodculture negative" in order to avoid any kind of byas according to the classification in "sepsis" and "contaminants". However, we agree with the reviewer that comparison of "sepsis" - "non-sepsis" is of interest and has included that kind of analysis in the results (page 10 line 22 and page 10 line 48). In summary these comparisons show the same trends as for the whole study group, however the numbers of included sepsisnewborns are limited, and the differences thus not statistically significant. - Our proportion of newborns with true CoNS sepsis however (6/46) however is similar to the incidence reported from other Western centra.

2. Why do we not present data on all 46 newborns? - In the present study we choose a casecontrol design and consider this as relevant, since only a minor proportion of the total cohort displayed a CoNS positive blood culture. However, we are just now analysing the data from all 46 preterms compared to healthy term newborns and plan to present these data in future manuscript.

3. Why do the findings of PFGE not quite match the $\mathrm{PhP}$ data? - A comment on this has been included in the Discusison (page 15 line 34).

4. Results of PFGE for typing validation of pairs of colonising and blood isolates identified as identical from PhP? - data has been included in the results (page 11 line 56).

5. Missing reference in the discussion. - Error in the proof by us. - The last part of the sentence that would correspond to the missing reference was thought to be omitted already in the original manuscript. This is now corrected.

We hope that the reviewer will find the new version of the manuscript improved compared to the original version and suggest it for publication in EJCMID. 